\title{
Relationship of Adult Attachment Styles and External Shame on Depression Symptoms among Married Couples: A Preliminary Study
}

\author{
Nor Ba'yah Abdul Kadir \\ Wan Shahrazad Wan Sulaiman \\ Asmawati Desa \\ Fatimah Omar \\ Fatimah wati Halim \\ Fatimah Yusooff \\ Universiti Kebangsaan Malaysia
}

Corresponding author:

Nor Ba'yah Abdul Kadir, PhD

aknbayah@ukm.edu.my

Psychology and Human Wellbeing Research Centre, Faculty of Social

Sciences and Humanities, Universiti Kebangsaan Malaysia, 43600 Bangi,

Selangor, MALAYSIA

Tel: +603-89213674; Fax: +603-89213541

\section{Abstract}

This study investigated the potential impact of attachment styles and external shame on depression symptoms among married couples from low-income apartments in the suburbs of Kuala Lumpur. A door-to-door approach was used to recruit participants and collect data. Three research scales were used in this study: the Experience in Close Relationship-Revised Version questionnaire was used to measure individual differences in adult attachment styles, the Other as Shamer Scale was used to measure external shame, and the Personal Health Questionnaire was used to measure depression symptoms. This study reports that the 
most common depression symptoms reported are among individuals who experienced depression symptoms for less than one month. Results showed that dualinsecure couples (wives' anxiety attachment and husbands' avoidance attachment, wives' avoidance attachment and husbands' avoidance attachment) were significantly correlated with depression in a sample of the wives. The findings also revealed that a specific type of dual-insecure couples (wives' anxiety attachment and husbands' avoidance attachment) was a predictor of depression symptoms. These results provide evidence for a role of specific dual-insecure couples and external shame on depression symptoms.

Keywords: dual-insecure relationships, mental health, vulnerability, Malaysia

A number of scholars have suggested that the quality of a couple's relationship is grounded in attachment style, the individual patterns being among the most important factors. Secure attachment has been identified as a major positive influence in explaining variations in the quality of good marital relationships (Molero et al., 2016; Strauss, Morry, \& Kito, 2012). What can we expect when one or both parties in the couple have anxiety attachment or avoidance attachment? Givertz and colleagues (2013) found that both anxious and anxiety attachments were associated with lower relationship quality and that one partner's insecure attachment was associated with the spouse's report of lower relationship quality. Other studies reported a sense of insecurity in one's current romantic relationship was significantly related to depression between partners, however, an individual's secure attachment reduced one's own depression (Mehta, Cowan, \& Cowan, 2009). These results indicated that couples' relationships clearly influence depression (Beach \& Whisman, 2012). While there is a clear association between depression and personal relationship, there is a need to identify the specific pattern of insecure attachment styles that influence this association (Heene, Buysse, \& Van Oost, 2005). Insecure attachment may be a significant factor that offers increased understanding the dynamics of coupled relationships, and particularly may influence depression (Jinyao, Xiongzhao, Auerbach, Gardiner, Lin, Yuping et al., 2012) and external shame (Gilbert \& Miles, 2000).

Research on the effects of adult anxiety attachment, avoidance attachment, and external shame on depression symptoms is scarce in close relationship studies. Only a few studies were found that investigated the impact of attachment styles and external shame on collaborative problem-solving orientations (Lopez, Gover, Leskela, Sauer, Schirmer, \& Wyssmann, 1997), the connection between attachment styles and investment in relatedness (Gross \& Hansen, 2000), associations of personality, shame, and social bonds (Shaher, 2001), adult attachment styles in relation to shame, depression, and loneliness (Wei, Shaffer, Young, \& Zakalik, 2005), and traumatic memory-related shame 
in relation to depression (Matos \& Pinto-Gouveia, 2014, 2013). For instance, Matos and Pinto-Gouveia (2014) examined the phenomenological characteristics of shame-related childhood memories and their impact on psychopathology among 230 adults in Portugal. The study revealed that shame memories showed a higher correlation between external shame and depression while higher correlations between internal shame and depression were seen in relation to attachment figures.

One study discovered that preoccupied and fearful attachment styles were significantly related to shame, while those with secure attachment style were less vulnerable to the experience of shame (Gross \& Hansen, 2000). Relating this to a working model of attachments (Bartholomew \& Horowitz, 1991), those with preoccupied attachment style and those with fearful attachment style demonstrated a negative sense of self that would lead to higher levels of experiencing shame. Therefore, those who have a high need to be loved and accepted, and who worry about hurting or offending others in a close relationship may be more sensitive and vulnerable to experience shame. Such concerns may cause some negative feelings, such as fear of rejection, separation, or feeling unwanted, all linked to shame. Thus, individuals with preoccupied and fearful styles increase doubt about their competency and lovability in a close relationship, and would report higher levels of shame that could lead to mental health problems.

Kilmann and colleagues (2012) studied partner attachment and interpersonal characteristics among 113 non-clinical couples in long-term marriages. They discovered that spouses with greater anxiety attachment endorsed dysfunctional relationship beliefs. The study also reported that husbands with higher scores for anxiety attachment were rated less aggressive, less controlling, and more rebellious; wives with a higher score in this dimension were rated more dependent, more self-critical, and less competitive. The study also revealed that greater marital distress more likely existed in marriages consisting of at least one insecure partner.

\section{The present study}

Although contributing factors and the negative impact of adult attachment styles on psychosocial functioning are well-established using retrospective and prospective designs in most Western countries, attachment styles among married couples have not yet been investigated in many Eastern countries. Studies concerning attachment styles among married couples have been lacking due to the time and costs involved, as well as the low response from the participants, especially male participants. This study aims to fill this research gap by investigating attachment styles among married couples in the Malaysian 
context. More precisely, this study examines whether the attachment style of married couples is significantly correlated with depression. It is our hope that this research can help fill a gap that has been present for decades in psychology literature concerning adult attachment styles and depression among married couples. The study's researchers reviewed close relationship studies from 2007 to 2014 using Google Scholar, ScienceDirect, and Ebscohost, and only found eight relevant studies (e.g., Melore, Shaver, Ferrer, Cuadrado, \& Alonso-Arbiol, 2010; Givertz, Woszidle, Sergin, \& Knutson, 2013; Monin, Feeney, \& Schulz, 2011). These studies were conducted among couples in the United States, Canada, and Spain. Thus, the present study has a threefold objective: First, this study aims to investigate the tabulation of duration for depression symptoms; second, to examine the relationships between anxiety attachment, avoidance attachment, and external shame of depression; and finally, to determine predictors of depression in a sample of married couple pairings in Malaysia.

\section{Method}

\section{Participants and Procedure}

This study was conducted though door-to-door surveys in a public housing residential neighborhood in Kuala Lumpur. Research materials were conducted in the Malay language as it is widely understood and spoken, and it is a national language of Malaysia. The primary sources of participant employment were government services, small local business ventures, and factory work.

Participant couples were married, on average, 16.60 years (S.D. 11.06). The mean age of the wives was 39.75 years old (S.D. 11.81), and the husbands was 44.41 years old (S.D. 12.67). The sample was comprised predominantly of Malays (71.9\%; $n=286)$, with 21.6 percent $(n=86)$ Indians, 5.0 percent $(n=20)$ Chinese, and others making up 1.5 percent $(n=6)$. Fewer than half of the wives were employed $(41 \%, \mathrm{n}=82)$ with a mean monthly income of $\$ 123.43$ (USD), and the rest were homemakers. In terms of education, 61.5 percent $(n=123)$ of the wives had completed a secondary education and 38.5 percent $(n=77)$ finished up to primary school. Most of the husbands were employed (87 percent, $n=174$ ) with a mean monthly income of $\$ 322.56$. In terms of education, 61 percent $(n=112)$ of the husbands had completed their secondary education and 39 percent had finished up to primary school. One married couple did not return their questionnaires; therefore, data for 199 married couples were available for analysis.

In the first phase of sampling selection, a total of 11 public housing areas under the administration of the Public Housing Programme in Kuala Lumpur were identified as our potential sample. Official letters were sent to the City 
Hall of Kuala Lumpur for research approval and collaboration. The sample then was derived from the Residential Community Association, and a total of 199 married couples completed the questionnaire. No access to the lists of names and addresses was provided by the community leaders due to confidentiality issues, therefore a systematic sampling was applied. However, due to the unexpected response rate, a convenience sampling and door-to-door distribution of questionnaires was applied. A complete description of the methods, sample selection, and respondents is provided in a separate article on this study (Abdul Kadir et al., 2017). To be eligible for participation in the study, we required that both participants within couples be in their first marriage, with a minimum marriage duration of two years, between the ages of 21 and 80 years old, have at least one child at home under the age of 17 years, and living in a suburb apartment under the authority of the Public Housing Programme in Kuala Lumpur. Married couples without children were not included, based on literature findings that childless couples were more satisfied in their marital relationship, less worried, experienced low levels of anxiety and depression, and were happier than married couples with children (Zhang \& Liu, 2007). In contrast, married couples with children may experience high and low emotions because of unrealistic expectations and a lack of agreement in regards to assumed gender roles (Eccles, 1987). This parallels with the attachment activation system, in that attachment style is activated when individuals experience stress or crisis.

Each couple was provided with a questionnaire pack. They were asked to complete the questionnaire alone. In this study, a research assistant and a group of undergraduate students in a social work programme (enumerators) introduced the study to potential participants, helped the respondents to answer the questionnaires, and gained verbal consent for inclusion in the study. A research assistant and enumerators further explained that all information would be coded and only accessible to the research team. The research assistant and enumerators administered a socio-demographic questionnaire along with the Malay translation of the Experience in Close Relationship-Revised Version (ERC), the Other as Shamer Scale (OAS), and the Personal Health Questionnaire (PHQ). The research assistant and enumerators read out the items of the questionnaire to the participants who were illiterate. The married couples took between 20 and 35 minutes to complete the questionnaire pack. Using this approach, 199 married couples returned completed questionnaires.

\section{Ethical Permission}

This study followed standard guidelines for conducting empirical research. Therefore, all participants provided informed consent with the assurance of confidentiality and anonymity. Approval was also sought from Kuala Lumpur City Hall (DBKL). 


\section{Measures}

All questionnaires were identical and assessed several domains including socio-demographic factors (e.g., religion, ethnicity, monthly income, level of education, number of children), psychosocial vulnerability factors (e.g., attachment styles, external shame), and depression symptoms. These standardized questionnaires were translated into the Malay language and translated back to English before use to ensure the accuracy of meaning with the first author and research team members. Discrepancies were discussed and the wording changed for ambiguous items, and it was translated back into Malay. Two independent professional translators translated the items, and following a cultural adaptation, a final version was completed.

\section{Attachment styles}

To assess the spouses' attachment styles, each spouse completed the self-report measure developed by Fraley, Waller, and Brennan (2000). This instrument was used to assess individual differences on two subscales of attachment styles: avoidance attachment and anxiety attachment. The participants responded on 5-point Likert scales to indicate how much the participants agreed with each statement about close relationships. The scores ranged from 1 (strongly disagree) to 5 (strongly agree) to endorse the items. A higher score on anxiety attachment indicates individuals tend to have fear of rejection and abandonment and a higher score on avoidance attachment indicates individuals find discomfort with intimacy and seek independency. In this study, the Cronbach's alpha for anxiety attachment was 0.86 . The published internal consistency of anxiety attachment was 0.86 . The Cronbach's alpha for avoidance attachment was 0.82 . The published internal consistency of avoidance attachment was 0.92 .

\section{External Shame}

To assess external shame, both husbands and wives completed a self-report measure developed by Goss, Gilbert, and Allan (1994). The scale was found to correlate highly with Internalized Shame Scale $(r=0.81)$, indicating that the scale can be used as a measure of shame proneness (trait shame) and moreover that shame involves both self and other evaluations (Goss et al., 1994). This scale consists of 18 items rated on a five-point scale according to the frequency of evaluation about how others judged themselves, $(0=$ Never to $4=$ Almost always). The Cronbach's alpha of this scale was 0.92 . The published internal consistency was 0.92 (Goss et al., 1994) and 0.95 (Cunha, Matos, Faria, \& Zagala, 2012). The OAS has been widely used in many studies (Gilbert \& Miles, 2000; Birchwood et al., 2007). 


\section{Depression symptoms}

To assess depression symptoms, both husbands and wives completed a selfreport measure developed by Goldberg and Simpson (1995, unpublished). Three items investigate the main depressive symptoms under criterion A of ICD-10 and seven items investigate additional depressive symptoms under criterion B of ICD-10. One further item was developed to measure the duration of depressive symptoms but does not contribute to the total scores. Each symptom is rated between 0 and 2 according to its occurrence over the previous two weeks (never; occasionally; most days), giving a total score of between 0 and 20. A Likert score $\leq 9$ would be considered as a presence of depressive disorders. The Cronbach's alpha of this scale was 0.88. A higher score indicates a greater experience of depression.

\section{Results}

The first analysis examined the distribution of duration for individual depression symptoms. Next, associations between adult attachment styles and external shame on depression symptoms were examined using Pearson's Correlation. Finally, multiple regression was applied to determine predictors of depression symptoms.

\section{Distribution of duration for depression symptoms}

Further questions were asked to determine whether participating married couples had more than four symptoms of depression, to assess the duration of the symptoms of depression (Table 1). The results suggest that the most common of the depression symptoms were among those who experienced symptoms for less than one month ( $\mathrm{n}=165 ; 41.5$ percent), followed by one month to one year ( $n=37 ; 9.3$ percent) and more than one year $(n=26 ; 6.5$ percent). The least common of the symptoms was more than one year but getting worse $(n=2 ; 0.05$ percent).

Table 1. Tabulation of duration for depressive symptoms for those having more than four depressive symptoms

\begin{tabular}{lcr}
\hline Variables & Husbands & Wives \\
\hline Less than 1 month & $\mathrm{N}(\%)$ & $\mathrm{N}(\%)$ \\
1 month to 1 year & $82(41.2)$ & $83(41.7)$ \\
More than 1 year & $17(8.5)$ & $20(10.1)$ \\
More than 1 year but getting worse & $12(6.0)$ & $13(6.5)$ \\
\hline
\end{tabular}


Correlation of attachment dimensions and external shame for depression symptoms

The correlation of anxiety attachment, avoidance attachment, and external shame among the sample of wives was positive, but not particularly strong. These patterns of correlation indicated that both attachments were positively related to depression symptoms (Table 2). However, no significant correlation was found in the sample of husbands for both attachment styles except for external shame. The pattern of correlations among husbands indicated that both attachment styles were unrelated to depression symptoms (Table 2).

Table 2. Correlation of adult attachment styles and external shame with depression symptoms

\begin{tabular}{lc}
\hline Variables & Depression symptoms \\
\hline Husbands & \\
Anxiety attachment & 0.08 \\
Avoidance attachment & -0.01 \\
External Shame & $0.35^{\star \star}$ \\
Wives & \\
Anxiety attachment & $0.18^{\star \star}$ \\
Avoidance attachment & $0.20^{\star \star}$ \\
External Shame & $0.45^{\star \star}$ \\
\hline
\end{tabular}

**. Correlation is significant at the 0.01 level

Correlations of the married couples' attachment dimension for depression symptoms

Further analysis was carried out to examine the correlations of married couples' attachment styles with depression symptoms (Table 3). The results showed that the wives with anxiety attachment married to avoidant husbands, and wives with avoidant attachment married to avoidant husbands showed a positive but weak correlation with depression symptoms (Table 3). These results indicated that wives with anxiety attachment married to avoidant husbands and those wives with avoidant attachment married to avoidant husbands would be likely to score high for depression symptoms.

\section{Predictors of depression in a sample of the wives}

Multiple regression analysis was employed to examine the predictors of depression. Before multiple regression analysis was undertaken, several requirements and statistical assumptions were checked. The requirements and assumptions comprised sample size and normality, homoscedasticity, linearity, multicol- 
Table 3. Correlation for depression symptoms

\begin{tabular}{lcc}
\hline & $\begin{array}{c}\text { Depression } \\
\text { (Wives) }\end{array}$ & $\begin{array}{c}\text { Depression } \\
\text { (Husbands) }\end{array}$ \\
\hline $\begin{array}{l}\text { 1. Wives anxiety attachment/husbands anxiety } \\
\text { attachment }\end{array}$ & 0.11 & -0.05 \\
$\begin{array}{l}\text { 2. Wives anxiety attachment/husbands avoidance } \\
\text { attachment }\end{array}$ & $0.14^{\star}$ & -0.11 \\
$\begin{array}{l}\text { 3. Wives avoidance attachment/husbands } \\
\text { avoidance attachment }\end{array}$ & $0.14^{\star}$ & 0.03 \\
$\begin{array}{l}\text { 4. Wives avoidance attachment/husbands } \\
\text { anxiety attachment }\end{array}$ & 0.12 & 0.09 \\
\hline
\end{tabular}

*. Correlation is significant at the 0.05 level (2-tailed)

linearity, and outliers. We examined these assumptions for multiple regression analysis and found that the sample size fulfilled the minimum requirement, as the present study included four main variables (anxiety attachment, avoidance attachment, external shame, and depression symptoms); the sample size of 199 was adequate according to recommendations by Hair and colleagues (2006) that ideal sample sizes contain between 5 and 20 respondents for each variable. The assumption of normal distribution was also fulfilled based on the graphical examination that showed that the data were distributed normally. The skewness and kurtosis values also fulfilled the minimum requirement. The assumption of homoscedasticity showed through assessment of the scatterplot that the variances were equal. Examination of the scatterplot graph showed that the assumption of linearity was fulfilled as the predictor and criterion data were distributed along a linear line.

Singularity was not violated as the predictor variables comprised independent and unique variables that did not overlap. In order to avoid the potential problems of overlapping between variables, multicollinearity tests were examined. Since all the tolerance indices were greater than $1-\mathrm{R}^{2}$, none of the study variables was removed. Subsequently, further analysis was undertaken to examine the model of predictors of depression symptoms. Using the stepwise method, a significant model emerged (Table 4). The model produced one predictor of depression symptoms in a sample of the wives, which contributed 14 percent of the variance (adjusted R square 0.021, F $(1,197)=4.19, \mathrm{p}<0.05$ ) on depression symptoms. As predicted, participant wives with anxiety attachment married to avoidant husbands were significantly correlated to depression symptoms to a certain degree. To be specific, wives with anxiety attachment married to avoidant husbands scored high in anxiety attachment and were found to have high depression symptoms. None of the other predictors was significantly correlated with depression symptoms. 
Table 4. Predictors of depression among wives

\begin{tabular}{llcc}
\hline Predictors & Beta & SE & Sig. \\
\hline $\begin{array}{l}\text { Wives anxiety attachment/husbands avoidance } \\
\text { attachment }\end{array}$ & 0.14 & 0.29 & .04
\end{tabular}

Note: Wives anxiety attachment/husbands anxiety attachment, Wives avoidance attachment/husbands avoidance attachment, and Wives avoidance attachment/husbands anxiety attachment were not predictors of depression

\section{Discussion and Conclusions}

Attachment styles, external shame, and their relation to depression symptoms were the focus of this study. The research team was interested whether specific patterns of insecure attachment pairings were more likely to be associated with depression symptoms in this sample of married couples. Finally, this study also sought to determine whether a specific pattern of insecure attachment in individual members of couples could predict depression symptoms.

In this study, more than half of the husbands and wives reported having more than four depressive symptoms. Our findings were not consistent with the findings of previous research (e.g., Kessler, McGonagle, Swartz, Blazer, \& Nelson, 1993; Rohde, Lewinsohn, Klein, Seeley, \& Gau, 2013) in terms of gender, where reports suggested males were more than twice as likely to experience depression symptoms as females. Our findings suggested that both men and women reported high rates of depression symptoms. The findings offered an important significant contribution to our understanding of adult depression symptoms, particularly those married couples with insecure attachment styles. We suggested that gender differences in depression symptoms may due to social roles occupied by men and women. Social roles may be increasingly different as the level of family commitment increases, for instance married women with children have an increased risk of depression symptoms due to multiple roles to perform. This can be also explained in terms of traditional roles as homemaker and caregiver may increase stress due to their responsibilities to manage children, elderly parents, and household chores. In similar employed wives with young children are also expected to perform their duties and responsibilities for the sake of family's well-being particularly when they do not earn sufficient amounts to hire out their caregiver duties. Previous studies reported that having more than four young children at home would increase the risk of depression symptoms (Brown \& Harris, 1978).

We found that the wives who reported higher anxiety attachment or greater avoidance attachment reported greater experience for depression, while no correlations were found among the husbands. These findings were consistent with the findings of other research that individuals with anxiety hold signifi- 
cantly more irrational beliefs (Stackert \& Bursik, 2003) and a negative evaluation of self (Bifulco \& Moran, 1998; Murphy \& Bates, 1997), which tends to be linked with mental disorder, particularly depression (Bifulco, Moran, Ball, \& Bernazzani, 2002). Thus, we suggested that those with higher scores on anxiety attachment or avoidance attachment or both associated with depression symptoms are more vulnerable and distressing. Relating our findings with attachment theory, we concluded that higher anxiety attachment and avoidance attachment were the most important factors in increased risks of depression.

Our findings suggested that wives with anxiety attachment and wives with avoidance attachment were significantly correlated with depression symptoms. The findings for wives suggest that additional processes may need to be considered in order to fully understand wives' depression symptoms. Additionally, this could likely create troubled close relationships with the husbands, increase marital conflicts, and, thus, would lead to depression symptoms. We also conclude that those wives with anxiety attachment were extremely emotionally sensitive with their avoidant husbands, which can lead to depression symptoms.

Our findings also suggest that external shame was also significantly related with depression symptoms. This finding is consistent with other findings (Balsamo et al., 2015; Gilbert \& Miles, 2000; Gilbert, McEwan, Bellew, Mills, \& Gale, 2009; Goss et al., 1994). We concluded that external shame emerged as a signal which insecure wives experienced fear of negative evaluation of others, such as unattractive, unworthy or unacceptable. In terms of the internal working models of self and others, it might be that respondent wives with high levels of anxiety attachment tended to have a negative working model of self, including experiencing the self as defective, unlovable, unworthy, undesirable in the mind of the spouse or attachment figures; this model sets others as threatening, harsh, hostile and powerful, and thus may have become part of a negative relational schema. Further, respondent wives with high levels of avoidance attachment were more likely to have a negative model of others and have learned that self-reliance was a preferable model. In addition, such wives tended to believe that others were unavailable to help them in times of stress. Therefore, they were more likely to rely on themselves in order to manage their stress. Another possible explanation is that the wives could find relationships with others particularly difficult due to their concern about being discovered as worthless and others losing interest. Our results were also in line with Lewis' (1971) suggestion that "shame involves the failure of the central attachment bond" whereas Kaufman (1989) relates shame to the experience of being emotionally and psychologically detached from attachment figures. Shame, whether internal or external, involves extreme inferiority, worthlessness, and unlovability that may be associated with the experiences of fears of being rejected and separated (Lopez et al., 1997).

In relating our findings with traditional gender roles in which the wives 
were expected to be obedient, passive, and devoted to their husbands; these values and practices would influence attachment styles. Because culture exerts a strong influence on both gender role practices and the way in which those practices are interpreted by the spouses, it is also reasonable to expect a somewhat differential distribution of attachments. This is in line with attachment theory that states the long history of interpersonal development and behaviors within the culture and social values would shape the variability in an attachment activation system. Thus, the frequency and quality of interactions and supportive relationship in times of crisis between spouses may form the basis of different attachments. Another potential reason that culture would shape attachments can be attributed to the nature of collective culture because collective individuals merge their identity with their society and prioritize the collective goals over their personal goals. For instance, Asians emphasize social harmony (Markus et al., 1997), fear of being on one's own, of being separated or disconnected from others (Markus \& Kitayama, 1994), and tend to suppress their own emotions (Butler, Lee, \& Gross, 2007) which would lead to avoidance attachment, meanwhile unable to maintain social harmony and trust may lead to fears of being rejected and abandoned, which, in turn, would shape anxiety attachment.

Our study is the first study in Malaysia that measured married couples in relation to depression symptoms. Our findings revealed that participant wives with anxiety attachment married to avoidant husbands reported that the most common depression symptoms; this was consistent with the findings of prior research (e.g., Scott \& Cordova, 2002). We also concluded that wives with anxiety attachment might be an important factor in increasing depression symptoms. We also suggested that the husband's avoidance attachment might affect one's own mental health. Thus, we suggested that the attachment styles of wives might be an important factor in shaping depression symptoms, while the husband's avoidance attachment can add to the depression symptoms. Future research should identify the risk factors that might help these couples to improve their marital satisfaction and intimacy in close relationships.

\section{Implications of the study}

Several implications can be drawn from this study. First, this study supports the development of adult attachment measures to be used in Malaysian society. This study adds to the existing literature concerning adult attachments and cultural issues, indicating support for a well-documented manual scoring and interpretation of anxiety and avoidance constructs. Support is also given for using adult attachment measures to be used in assessing and understanding the adult attachments among Malaysians. The findings of this study further highlight the roles of social service professionals, such as psychologists and 
counselors, to assess and understand the close relationship patterns of married couples before conducting marital therapy or counseling treatment. It is essential to note that adult attachment among Asian populations are different from Western people in that the attachment varies based on the region of origin, collectivism, and ethnicity (Agishtein \& Brumbaugh, 2013). In addition, Giudice (2011) discovered that East Asian males showed a particular pattern of avoidance attachment but no gender differences in anxiety attachment. This is in line with other studies that reported that Asian respondents reported higher levels of avoidance attachment than other secure and insecure attachments because this close relationship is heavily dependent on the opinion and expectation of others (Schmitt et al., 2003, 2004).

\section{Limitations}

This study acknowledges several limitations. First, our study relied on purposive sampling samples among public housing residents in the inner city of Kuala Lumpur, Malaysia. The Chinese and Indian samples were underrepresented when compared to the ratio of the racial composition of the population. Childless married couples were excluded and because of the small sample size due to poor response rates from other ethnic groups (e.g., Indian and Chinese), generalization for this study is restricted. Second, the adult attachment measure used in this study was a self-report measure, and, hence, the association of variables could be artificially inflated by the response style. Third, no early life measures were included to develop further the attachment themes in the genesis of the secure style. Future research is recommended to carry out further investigation of adult attachment styles and psychosocial factors, and their relationship with depression symptoms among rural Malaysians. Despite the limitations, this study is able to discern the specific associations of couple pairings attachment on depression symptoms in the Malaysian context. Further research is needed that uses mixed measures on broader samples with extended measures of parenting self-efficacy, marital adjustment, resilience, and coping skills.

This study is likely to be the first to examine married couples in relation to adult attachments, external shame, and depression symptoms in the Malaysian context. Although our study provided some information about couple attachments, these findings should be considered preliminary. Despite certain limitations, our findings support the argument that the dimensions for matched and mismatched couple attachments need to be considered when studying adult attachments in relation to depression symptoms. Additional studies are required to confirm the associations between couple attachment, external shame, and depression by exploring four types of adult attachment style. Longitudinal studies are needed to explore the causal relationship between adult attach- 
ments and depression symptoms and the stability of adult attachments over the lifespan among married couples.

\section{References}

Abdul Kadir, N. B., Wan Sulaiman, W. S., Desa, A., Omar, F., Halim, F. W., \& Yusooff, F. (2017). A Study on Attachment Orientations of Married Couples Living in the Suburbs of Kuala Lumpur and Rural Areas of Kota Bahru, Kelantan, Malaysia. The Family Journal, 25(2), 137-145. https://doi.org/10.1177/1066480717697969

Agishtein, P., \& Brumbaugh, C. (2013). Cultural variation in adult attachment: The impact of ethnicity, collectivism, and country of origin. Journal of Social, Evolutionary, and Cultural Psychology, 7, 384-405. https://doi.org/10.1037/h0099181

Beach, S. R., \& Whisman, M. A. (2012). Affective disorders. Journal of Marital and Family Therapy, 38(1), 201-219. https://doi.org/10.1111/j.1752-0606.2011.00243.x

Bifulco, A., Brown, G. W., Moran, P., Ball, C., \& Campbell, C. (1998). Predicting depression in women: the role of past and present vulnerability. Psychological Medicine, 28(1), 39-50. https://doi.org/10.1017/S0033291797005953

Bifulco, A., Moran, P. M., Ball, C., \& Bernazzani, O. (2002). Adult Attachment Style I: Its relationship to clinical depression. Social Psychiatry \& Psychiatric Epidemiology, 37, 50-59. https://doi.org/10.1007/s127-002-8215-0

Birchwood, M., Trower, P., Brunet, K., Gilbert, P., Iqbal, Z., \& Jackson, C. (2007). Social anxiety and the shame of psychosis: A study in first-episode psychosis. Behaviour Research and Therapy, 45, 1025-1103. https://doi.org/10.1016/j.brat.2006.07.011

Brown, G. W., \& Harris, T. (1978). Social origins of depression. London: Tavistock Publications.

Cunha, M., Matos, M., Faria, D., \& Zagala, S. (2012). Shame memories and psychopathology in adolescence: The mediator effect of shame. International Journal of Psychology \& Psychological Therapy, 12, 203-218.

Eccles, J. S. (1987). Gender roles and women's achievement-related decisions. Psychology of Women Quarterly, 11, 135-172. https://doi.org/10.1111/j.1471-6402.1987. tb00781.x

Butler, E. A., Lee, T. L., \& Gross, J. J. (2007). Emotion regulation and culture: are the social consequences of emotion suppression culture-specific? Emotion, 7(1), 30. https://doi.org/10.1037/1528-3542.7.1.30

Gilbert, P., \& Miles, J. N. (2000). Sensitivity to Social Put-Down: Its relationship to perceptions of social rank, shame, social anxiety, depression, anger and self-other blame. Personality and individual differences, 29, 757-774. https://doi.org/10.1016/ S0191-8869(99)00230-5

Giudice, M. D. (2011). Sex differences in romantic attachment: A metaanalysis. Personality and Social Psychology Bulletin, 37, 193-214. https://doi. org/10.1177/0146167210392789

Givertz, M., Woszidlo, A., Segrin, C., \& Knutson, K. (2013). Direct and indirect effects of attachment orientation on relationship quality and loneliness in married couples. Journal of Social and Personal Relationships, 30, 1096-1120. https://doi. org/10.1177/0265407513482445

Goss, K., Gilbert, P. \& Allan, S. (1994) An exploration of shame measures: I: The 'other 
as shamer'scale. Personality and Individual Differences, 17, 713-717. https://doi. org/10.1016/0191-8869(94)90149-X

Heene, E. L. D., Buysse A., \& Oost, P. V. (2005). Indirect pathways between depressive symptoms and marital distress: The role of conflict communication, attributions, and attachment style. Family Process, 44, 413-440. https://doi.org/10.1111/j.15455300.2005.00070.x

Jinyao, Y., Xiongzhao, Z., Auerbach, R. P., Gardiner, C. K., Lin, C., Yuping, W., et al. (2012). Insecure attachment as a predictor of depressive and anxious symptomology. Depression and Anxiety, 29, 789-796. https://doi.org/10.1002/da.21953

Kaufman, G. (1989). The psychology of shame: Theory and treatment of shame-based syndromes. New York: Springer.

Kessler, R. C., McGonagle, K. A., Swartz, M., Blazer, D. G., \& Nelson, C. B. (1993). Sex and depression in the National Comorbidity Survey I: Lifetime prevalence, chronicity, and recurrence. Journal of Affective Disorders, 29, 85-96. https://doi. org/10.1016/0165-0327(93)90026-G

Kilmann, P. R., Finch, H., Parnell, M. M., \& Downer, J. T. (2012). Partner attachment and interpersonal characteristics. Journal of Sex and Marital Therapy, 38, 1-16.

Lewis, H. B. (1971). Shame and guilt in neurosis. New York, NY: International Universities Press.

Lopez, F. G., Gover, M. R., Leskela, J., Sauer, E. M., Schirmer, L., \& Wyssmann, J. (1997). Attachment styles, shame, guilt, and collaborative problem-solving orientations. Personal Relationships, 4, 187-199. https://doi.org/10.1111/j.1475-6811.1997. tb00138.x

Markus, H. R., \& Kitayama, S. (1994). The cultural construction of self and emotion: Implications for social behavior. In S. Kitayama \& H. R. Markus (Eds.), Emotion and culture: Empirical studies of mutual influence (pp. 89-132). Washington, DC: American Psychological Association. https://doi.org/10.1037/10152-003

Markus, H. R., Mullally, P. \& Kitayama, S. (1997). Selfways: Diversity in modes of cultural participation. In U. Neisser \& D. A. Jopling (Eds.), The conceptual self in context: Culture, experience, self-understanding (pp. 13-61). Cambridge, England: Cambridge University Press.

Matos, M., \& Pinto-Gouveia, J. (2014). Shamed by a parent or by others: The role of attachment in shame memories relation to depression. International Journal of Psychology and Psychological Therapy, 14, 217-244.

Matos, M., Pinto-Gouveia, J., \& Costa, V. (2013). Understanding the importance of attachment in shame traumatic memory relation to depression: The impact of emotion regulation processes. Clinical Psychology \& Psychotherapy, 20(2), 149-165. https://doi.org/10.1002/cpp.786

Mehta, N., Cowan, P. A., \& Cowan, C. P. (2009). Working models of attachment to parents and partners: Implications for emotional behavior between partners. Journal of Family Psychology, 23, 895-899. https://doi.org/10.1037/a0016479

Molero, F., Shaver, P. R., Fernandez, I., Alonso-Arbiol, I., \& Recio, P. (2016). Long-term partners' relationship satisfaction and their perceptions of each other's attachment insecurities. Personal Relationships, 23(1), 159-171. https://doi.org/10.1111/ pere. 12117

Monin, J. K., Feeney, B. C., \& Schulz, R. (2012). Attachment orientation and reactions to anxiety expression in close relationships. Personal Relationships, 19, 535-550. https://doi.org/10.1111/j.1475-6811.2011.01376.x 
Murphy, B., \& Bates, G. W. (1997). Adult attachment style and vulnerability to depression. Personality and Individual Differences, 22, 835-844. https://doi.org/10.1016/ S0191-8869(96)00277-2

Rohde, P., Lewinsohn, P. M., Klein, D. N., Seeley, J. R., \& Gau, J. M. (2013). Key characteristics of major depressive disorder occurring in childhood, adolescence, emerging adulthood, and adulthood. Clinical Psychological Science, 1, 41-53. https://doi. org/10.1177/2167702612457599

Schmitt, D. P. (2003). Are men universally more dismissing than women? Gender differences in romantic attachment across 62 cultural regions. Personal Relationships, 10, 307-331. https://doi.org/10.1111/1475-6811.00052

Schmitt, D. P., Alcalay, L., Allensworth*, M., Allik, J., Ault, L., Austers, I., et al. (2004). Patterns and universals of adult romantic attachment across 62 cultural regions: Are models of self and of other pancultural constructs? Journal of Cross-Cultural Psychology, 35, 367-402. https://doi.org/10.1177/0022022104266105

Scott, R. L., \& Cordova, J. V. (2002). The influence of adult attachment styles on the association between marital adjustment and depressive symptoms. Journal of Family Psychology, 16, 199-208. https://doi.org/10.1037/0893-3200.16.2.199

Stackert, R. A., \& Bursik, K. (2003). Why am I unsatisfied? Adult attachment style, gendered irrational relationship beliefs, and young adult romantic relationship satisfaction. Personality and Individual Differences, 34, 1419-1429. https://doi. org/10.1016/S0191-8869(02)00124-1

Strauss, C., Morry, M. M., \& Kito, M. (2012). Attachment styles and relationship quality: Actual, perceived, and ideal partner matching. Personal Relationships, 19(1), 14-36. https://doi.org/10.1111/j.1475-6811.2010.01333.x

Wei, M., Shaffer, P. A., Young, S. K., \& Zakalik, R. A. (2005). Adult attachment, shame, depression, and loneliness: The mediation role of basic psychological needs satisfaction. Journal of Counseling Psychology, 52, 591-601. https://doi.org/10.1037/00220167.52.4.591

Zhang, W., \& Liu, G. (2007). Childlessness, psychological well-being, and life satisfaction among the elderly in China. Journal of Cross-cultural Gerontology, 22(2), 185203. https://doi.org/10.1007/s10823-007-9037-3

\section{Acknowledgements}

This study was sponsored by the Ministry of Higher Education of Malaysia and Universiti Kebangsaan Malaysia, Grant UKM-SK-05-FRGS0227-2010. We thank the deputy director of the Housing Management \& Community Development Department of Kuala Lumpur City Hall who collaborated with this study, as well as the research assistants and social work undergraduate students who undertook the fieldwork and collected the data. 